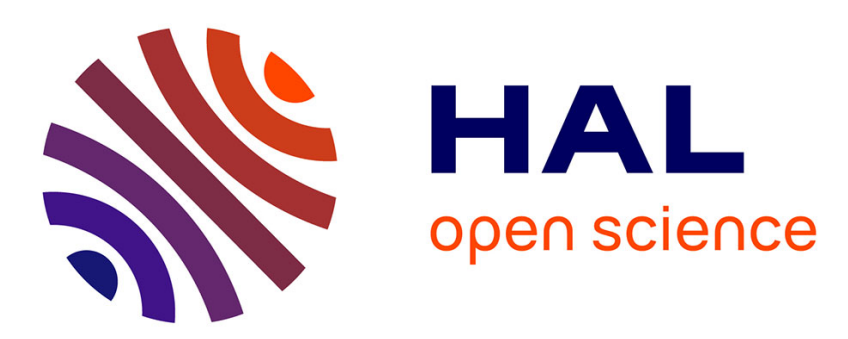

\title{
Le Sud-Soudan en paix? Sociologie politique d'une promesse d'indépendance
}

\author{
David Ambrosetti
}

\section{To cite this version:}

David Ambrosetti. Le Sud-Soudan en paix? Sociologie politique d'une promesse d'indépendance: Introduction au dossier "Conquérir l'indépendance, négocier l'État". Politique africaine, 2011, juin 2011 (122), pp.5-20. 10.3917/polaf.122.0005 . halshs-00617878

\section{HAL Id: halshs-00617878 \\ https://shs.hal.science/halshs-00617878}

Submitted on 20 Sep 2019

HAL is a multi-disciplinary open access archive for the deposit and dissemination of scientific research documents, whether they are published or not. The documents may come from teaching and research institutions in France or abroad, or from public or private research centers.
L'archive ouverte pluridisciplinaire HAL, est destinée au dépôt et à la diffusion de documents scientifiques de niveau recherche, publiés ou non, émanant des établissements d'enseignement et de recherche français ou étrangers, des laboratoires publics ou privés. 


\section{Coordonné par David Ambrosetti}

Introduction au thème

Le Sud-Soudan en paix ? Sociologie politique d'une promesse d'indépendance

"Mais y a-t-il deux peuples incapables de s'entendre ou trois ou dix ou mille ?"

H. Bleuchot, C. Delmet et D. Hopwood, «Introduction », in les mêmes, Sūdān. Histoire, identités, idéologies, Aix en Provence, IREMAM; St Antony's Middle East Monographs No. 25; Reading, Ithaca Press, 1991, p. x.

Sauf coup de théâtre, le Sud-Soudan accédera à l'indépendance en ce mois de juillet 2011. Difficile de ne pas y voir l'épilogue (pour les plus optimistes) de luttes politiques et armées engagées dès 1946, lorsque les autorités britanniques en charge du condominium angloégyptien se lancèrent dans la décolonisation d'un Soudan unifié, alliant Nord et Sud, pour prendre à revers la monarchie égyptienne et ses projets d'union avec le Soudan ${ }^{1}$. La demande d'indépendance du Sud-Soudan lancée par le parti libéral sudiste dès l'indépendance du Soudan en 1956 n'a-t-elle pas finalement trouvé un écho favorable auprès de la « communauté internationale ${ }^{2} »$ ?

Pendant près de soixante ans, la sécession a donc constitué une promesse, un horizon, pour les Sud-Soudanais. Une sociologie politique, et historique, de ce projet sécessionniste et de ses usages conduit à identifier plusieurs espaces dans lesquels s'est dessinée la trajectoire de ce projet. Les relations nouées avec les États de la région et sur la scène internationale ont ici pesé, en particulier dans la sphère d'influence anglo-américaine, de loin la plus intéressée par cette situation - n'est-ce pas d'ailleurs à cela que l'on doit la bizarrerie du nom français du nouvel État en passe de naître ${ }^{3}$ ? Mais l'élan essentiel se situe dans le face-à-face entre forces sudistes et nordistes. Depuis 1983, le gouvernement de la République du Soudan aujourd'hui dominé par le Parti du Congrès national (al muttamar al-wattani, PCN) - et les rebelles sudistes de l'Armée/Mouvement populaire de libération du Soudan (A/MPLS) s'opposent. De face-à-face, il n'y en eut finalement qu'assez peu, toutefois : la guerre sudsoudanaise, comme d'autres guerres écrites dans le langage de l'insurrection et de la contreinsurrection, a très tôt plongé ses ramifications au cœur des divisions internes, multipliant les relations croisées, et poussant la rébellion sudiste à opérer des choix de "gouvernement» face à la diversité et au conflit entre groupes sudistes rivaux. Ces choix ont laissé leur empreinte dans la façon dont ce nouvel État au Sud s'édifie, se négocie. L'actualité le montre aujourd'hui crûment.

C'est cet État en cours de négociation, entre différents ordres politiques qui s'entrechoquent aujourd'hui localement et sur la scène régionale, que nous avons choisi de présenter ici.

\section{De la guerre à la paix... à la guerre ?}

1. Voir D. H. Johnson, The Root Causes of Sudan's Civil Wars, Oxford, James Currey, 2003.

2. J. Oduho et W. Deng, The Problem of the Southern Sudan, Londres, Oxford University Press, 1963, p. 32.

3. Que ceux qui s'irritent du succès de cet anglicisme de la forme "Sud-Soudan " (comme "Sud-Vietnam» hier, une forme que le français n'utilise pas spontanément, préférant, par exemple, la Corée du Sud à la SudCorée) nous pardonnent : cette dénomination l'a emporté, y compris en France. Voir l'article publié sur le blog des correcteurs du Monde, «Soudan sud du Soudan du Sud-Soudan », 17 février 2011, $<$ correcteurs.blog.lemonde.fr>. Merci à Paul Guyonnet pour ses indications. 
Évoquer le Sud-Soudan aujourd'hui, c'est inviter le lecteur à plonger dans le feu de l'actualité la plus immédiate. Actualité d'une sécession annoncée, tout d'abord, conformément à l'accord de paix global (CPA) scellé à Naivasha, près de Nairobi, en janvier 2005, entre les deux principaux acteurs de la guerre en cours depuis 1983. Cette concession phare de la part des autorités soudanaises, portée alors par le vice-président du Soudan, Ali Osman Mohamed Taha, aboutit au scrutin de janvier 2011. Organisé avec le soutien conséquent d'acteurs internationaux, il a permis aux électeurs sud-soudanais d'exprimer massivement leur préférence en faveur de l'indépendance du Sud, ou plus exactement du territoire que Nordistes et Sudistes s'accordent aujourd'hui à reconnaître à ces derniers (à l'exclusion des zones demeurant litigieuses). Depuis la tenue du référendum, les regards se tournent vers les territoires situés en zone frontalière et dont l'avenir politique reste à déterminer : l'État du Nil bleu, l'État du Sud-Kordofan et, au sud de ce dernier, la zone d'Abyei. Cette dernière suscite depuis quelques mois une forte crispation de part et d'autre ${ }^{4}$, étant donné les enjeux pétroliers dans ces terres de pâture fertiles, très contestées localement.

Le Sud-Soudan occupe donc le devant de la scène du fait des sérieuses menaces qui pèsent sur le processus actuel de sécession et, au-delà, sur la paix et la sécurité dans la sous-région. Depuis 2010, un feu nourri de rapports alarmants s'abat sur les acteurs internationaux intéressés par la région, reflet de l'importance accordée par ces derniers à la situation du Soudan post-CPA. Cette situation atteint peut-être son paroxysme depuis le 21 mai dernier, alors que se sont intensifiés les combats entre forces gouvernementales et troupes de l'APLS (dans sa branche Nord) à Abyei et dans une large partie de l'État du Sud Kordofan (dont sa capitale Kadugli), produisant des dizaines de milliers de déplacements forcés, et que les combats menacent de s'étendre dans l'État du Nil bleu. Reste à voir si les accords obtenus, les 20 juin puis 28 juin à Addis-Abeba, grâce aux efforts du panel de haut niveau de l'Union africaine et de son chef Thabo Mbeki, proposant pour Abyei un retrait des deux forces en présence et le déploiement de forces d'interposition éthiopiennes, ainsi qu'une cessation des combats dans les deux autres zones de conflits, suffiront à contenir les tensions.

Parallèlement, les activités armées rebelles contre le gouvernement sud-soudanais se sont intensifiées à la fin du référendum, dans différentes zones pétrolifères dans les États du HautNil et de Jonglei ${ }^{5}$, même s'il semble que les rebelles s'appliquent à ne pas endommager les infrastructures pétrolières au cours des combats ${ }^{6}$. Les irrégularités qui ont marqué les élections générales organisées en 2010 au Soudan avaient conduit des candidats malheureux (George Athor, David Yauyau et Gatluak Gai), qui avaient quitté le MPLS pour concourir en tant qu'indépendants, à opter pour la confrontation armée contre le gouvernement sudiste dans les États de l'Unité et de Jonglei. Ces combats auraient tué plus de mille personnes, et auraient conduit au déplacement de dizaines de milliers en raison des violences commises par les forces en présence (APLS comprise) contre les civils ${ }^{7}$.

Parce qu'ils entrent dans le club des États souverains sous la double menace d'une plongée dans la guerre civile en différents endroits du pays et d'un retour à la confrontation généralisée avec l'ancienne tutelle au Nord, les Sud-Soudanais ne semblent pas promis à un avenir très enviable à court terme. D'aucuns parmi les observateurs internationaux présents sur le terrain disent même pouvoir écrire le parfait traité pour bâtisseurs d'État "faillis », mort-nés, à partir de cette expérience sud-soudanaise. On le verra dans le texte de Roland

4. « No Recognition for South Sudan if It Claims Abyei : Bashir », AFP, 29 avril 2011.

5. International Crisis Group, "Politics and Transition in the New South Sudan », Africa Report, $\mathrm{n}^{\circ} 172,4$ avril 2011 ; Human Rights Watch, Southern Sudan: Abuses on Both Sides in Upper Nile Clashes Hold Soldiers Accountable, Address Underlying Grievances, Juba, 19 avril 2011.

6. « Border Towns Feel like Pawns in Sudan Leader's Push for Oil », International Herald Tribune, 7 juin 2011.

7. «Update 1 - South Sudan Launches Attack, Kills 84 Rebels : Minister », Reuters, 11 mai 2011 ; «About 34,000 People Flee South Sudan Tribal Clashes : U.N. », Reuters, 6 avril 2011. 
Marchal dans ce dossier, le parallèle avec la sécession érythréenne et le regard porté sur l'environnement régional du Sud-Soudan conduisent à relativiser - mais non à invalider - ce jugement pessimiste, pour qui s'attache à comprendre les voies souvent originales par lesquelles des régimes assurent leur survie dans des contextes hostiles ${ }^{8}$. Il ne faudrait donc pas feindre de s'étonner des défis armés auxquels le MPLS fait face et de sa manière d'y répondre ; ils renvoient aussi à des histoires localisées anciennes, faites de complémentarités mais aussi d'accès de violences ${ }^{9}$. Certains appliqueront à l'ensemble du Soudan ce que Gérard Prunier a pu écrire à propos des relations intertribales au Darfour : que "le substrat des relations locales profondes, fondées sur l'écologie, l'histoire et les rapports ethniques prédétermine tout", ce qui n'exclut pas que "les vieilles relations de complémentarité reviennent tout aussi facilement"10.

Cela n'enlève rien à l'intense activité militaire actuellement en cours de toutes parts, au Sud mais aussi au Darfour ${ }^{11}$, et le vraisemblable activisme des services de sécurité soudanais aux côtés de ces rébellions sudistes, alors que la date de la sécession (le 9 juillet au plus tard) approche et que les arrangements pour le partage de la manne pétrolière et des autres ressources frontalières (comme les eaux du Nil) peinent à être trouvés. Du fait de la forte interdépendance existant entre les deux États, le retour à la confrontation ouverte constitue une option trop coûteuse pour les deux parties pour être envisagée à la légère. Mais Khartoum semble décidé à obtenir par différents leviers de pression le maintien des revenus du pétrole au niveau actuel, qui alimente le faste dans lequel vit la bourgeoisie de la capitale - peut-être une question de survie politique pour le président Béchir ${ }^{12}$.

\section{La sécession du Sud, " grâce " au Nord}

Paradoxalement, les autorités nord-soudanaises pourraient figurer parmi les meilleurs promoteurs du projet sécessionniste sudiste. La compétition politique à Khartoum, parce qu'elle a nourri la surenchère dans l'affirmation de l'arabité et de l'islamité du Soudan et de ses institutions politiques, a en effet servi de puissant catalyseur à l'argument sécessionniste au Sud. Au sortir de la Seconde Guerre mondiale, alors que se prépare l'indépendance du Soudan, l'establishment nord-soudanais promet au Sud une "soudanisation " censée rapprocher deux trajectoires historiques très différentes, en partie du fait de la politique britannique de développement séparé promouvant, au Sud, un «schéma de constitution d'unités tribales autosuffisantes fondées sur l'organisation et les coutumes indigènes ${ }^{13}$ » contre l'influence du Nord. Lors d'une première conférence tenue à Juba en 1947, des Sudistes s'inquiètent de voir le futur ex-colonisateur confier à l'État soudanais à venir les rênes des structures coloniales que le premier a édifiées dès 1902 pour administrer le SudSoudan séparément du Nord. Ces représentants réclament l'indépendance pour le Sud ou, à

8. C. Clapham, Africa and the International System. The Politics of State Survival, Cambridge, Cambridge University Press, 1996 ; P. Englebert et D. M. Tull, « Post-conflict reconstruction in Africa. Flawed Ideas about Failed States », International Security, vol. 32, n 4, 2008, p. 106-139.

9. C'est également le type d'analyse que propose Lise Grande, adjointe du coordinateur résident et coordinateur humanitaire des Nations unies au Sud-Soudan, à Reuters à propos des violences dans le Jonglei en février-mars 2011. «About 34,000 People Flee South Sudan Tribal Clashes : U.N. », Reuters, 6 avril 2011.

10. H. Bleuchot, C. Delmet et D. Hopwood, "Introduction », in les mêmes, Südān. Histoire, identités, idéologies, Aix en Provence, IREMAM; St Antony's Middle East Monographs No. 25; Reading, Ithaca Press, 1991, p. x, et p. 100-101 pour la citation tirée de la contribution de G. Prunier dans le même ouvrage.

11. « Fighting, Rights Abuses Escalating in Darfur : HRW », AFP, 7 juin 2011.

12. Border Towns Feel like Pawns in Sudan Leader's Push for Oil », International Herald Tribune, 7 juin 2011.

13. R. Gray, «Introduction », in J. Oduho et W. Deng, The Problem..., op. cit., p. 3.Voir également M. W. Daly, Empire on the Nile, Cambridge, Cambridge University Press, 1986. 
tout le moins, l'adoption de dispositions constitutionnelles garantissant une autonomie dans l'ensemble soudanais. La mutinerie générale du 18 août 1955, par laquelle débutent près de vingt ans d'insurrection armée menée par les rebelles de l'Anya Nya dans le Bahr el-Ghazal, l'Equatoria et une partie du Haut-Nil, chasse les représentants de Khartoum - sauf à Juba, dont la place ambivalente, tantôt garnison militaire de l'armée soudanaise, tantôt capitale administrative d'une région sud-soudanaise autonome après l'accord de paix signé à AddisAbeba en 1972, sert à la fois de cadre et d'énigme à l'article de Naseem Badiey dans ce dossier.

En novembre 1958, quand le Premier ministre Abdallah Khalil demande à l'armée et au général Abboud de prendre le pouvoir face au risque d'alliance entre forces ethno-régionales centrifuges, alors que les revendications sudistes font des émules en pays béja, la « coalition sociale hégémonique » qui gouverne à Khartoum montre combien elle reste rivée à l'affirmation politique de son arabité et de son islamité ${ }^{14}$. La guerre s'installe, tandis que Saturino Lohure, Joseph Oduho et William Deng, en exil, créent en 1964 l'Union nationaliste africaine du Soudan (SANU en anglais) pour mobiliser les soutiens africains contre Khartoum. Après l'accord de paix de 1972 voulu par le régime militaire de Jaafar Nemeyri (qui se saisit du pouvoir en 1969), proche de la gauche laïque, c'est Hassan al-Tourabi, héraut de l'islam politique des Frères musulmans, qui relance la course politique et symbolique à l'islamité, avec son Front national islamique (FNI). Sous sa pression, Nemeyri conteste les compétences précédemment octroyées aux Sudistes, et soutient une nouvelle campagne d'islamisation du Sud et les projets agricoles financés par des investissements de banques islamiques (avec la caution ultérieure du Fonds monétaire international) ${ }^{15}$.

La nouvelle guerre débute avec la mutinerie de Bor en mai 1983, puis les attaques armées contre les installations destinées à l'exploration pétrolière, alors que la charia est proclamée au Sud en septembre 1983. Comme en 1958, la perspective d'une remise en cause des fondements religieux et culturels de l'ordre politique soudanais poussent un régime civil à favoriser un coup d'État. En juin 1989, une coalition de forces nordistes et sudistes, civiles et militaires, soutient l'initiative de paix négociée à Addis-Abeba en novembre 1988, prévoyant notamment l'abrogation de la charia. Sadiq al-Mahdi, leader du parti de l'Oumma devenu Premier ministre en 1986, choisit de s'allier à son rival et beau-frère, Hassan Tourabi, leader du FNI, au nom de la protection des principes politiques islamiques. Marginalisé dans son projet d'instauration d'un ordre islamiste, le FNI mobilise ses relais dans l'armée soudanaise pour fomenter le coup d'État qui installe Omar Hassan el-Béchir au pouvoir à Khartoum au mois de juin $1989^{16}$ - avec pour conséquence une reprise du conflit armé au Sud sur le modèle anti-insurrectionnel précédent, accompagné d'une répression féroce des opposants nordistes. La politique contre-insurrectionnelle des autorités soudanaises (tant civiles que militaires), destinée à noyer l'APLS de John Garang dans les rivalités inter-sudistes, aurait certes pu atteindre son objectif ${ }^{17}$, mais elle a vraisemblablement renforcé la position pro-sécessionniste dans les rangs des combattants sudistes de différentes obédiences. Comparé à cette large adhésion au projet sécessionniste, le slogan de John Garang en faveur d'un « New Sudan »

\footnotetext{
14. Issues d'un petit nombre de familles riveraines du Nil, les élites soudanaises regardent vers le monde arabe et se reconnaissent dans les deux grandes confréries soufies (al-Mahdiya et al-Khatmyia) autour desquelles se polarise l'espace politique soudanais dès 1945 (avec respectivement le Parti Oumma et le Parti démocratique unioniste). Voir E. Ahmed. L'Élite du pouvoir au Soudan. Hégémonie et recrutement politique (1985-2000), thèse de doctorat en science politique, Université Montesquieu-Bordeaux IV, 2004.

15. Voir T. Barnett et A. Abdelkarim (dir.), Sudan : State, Capital, and Transformation, Londres, Croom Helm, 1988.

16. Voir E. Ahmed, «L'islam politique au Soudan. Les islamistes à l'épreuve de la direction de l'État (19892004) », in R. Otayek et B. Soares (dir.), Islam, État et société en Afrique, Paris, Karthala, 2009, p. 306.

17. Voir A. el-Battahani, «A Complex Web : Politics and Conflict in Sudan », Accord, $\mathrm{n}^{\circ} 18,2006$, disponible sur $<$ c-r.org $>$.
} 
uni et démocratique bénéficiant à l'ensemble des groupes ethno-régionaux soudanais a d'ailleurs constitué une anomalie - en grande partie guidée par des choix tactiques conjoncturels dus à l'hostilité du principal parrain étranger du mouvement rebelle (le régime du Derg à Addis-Abeba) à l'égard de toute revendication sécessionniste ${ }^{18}$. En réaction, le soutien apporté au cours des années 1990 par les autorités soudanaises à l'APLS-Nasir de Riek Machar et Lam Akol, née de la scission au sein du groupe rebelle en 1991, a été jusqu'à amener Khartoum à reconnaître le principe d'autodétermination du Sud par référendum dans la Charte pour la paix que les deux parties signent en $1997^{19}$.

Enfin, avec l'ouverture des négociations de paix entre l'APLS et Khartoum en 2001-2002, sous l'égide de l'Autorité intergouvernementale pour le développement (Igad) basée à Djibouti $^{20}$, et sous l'impulsion de l'administration américaine, s'ouvre une ère marquée par une congruence d'intérêts - toute conjoncturelle - de plus en plus forte entre les deux partenaires des accords de paix de Machakos (juillet 2002) puis de Naivasha (le CPA de janvier 2005). Le MPLS se détourne alors de ses alliances d'hier avec l'opposition soudanaise réunie au sein de l'Alliance démocratique nationale en exil à Asmara. Après la mort de John Garang dans un accident d'hélicoptère en juillet 2005, le mouvement semble habité par un seul objectif : saisir coûte que coûte cette opportunité unique d'accéder à l'indépendance du Sud-Soudan et, dans le même mouvement, d'installer le MPLS en situation hégémonique dans le futur État. L'ironie de l'histoire veut que le parti du Congrès national constitue probablement le meilleur allié politique du MPLS pour l'imposition de choix institutionnels favorables à ce dernier au sein du gouvernement autonome du Sud-Soudan (créé par l'accord de 2005). En échange, est attendue une solidarité (au moins implicite) du MPLS à l'égard du PCN - son associé gouvernemental à Khartoum - concernant les affaires politiques nordsoudanaises, lorsqu'il s'agira par exemple de défendre la charia au Nord ${ }^{21}$, ou les relations avec l'extérieur.

Le CPA octroie $70 \%$ des postes de l'exécutif et du législatif au MPLS dans le gouvernement du Sud-Soudan, mais aussi dans les institutions des dix États fédérés composant le SudSoudan. De fait, le MPLS occupe, en avril 2011, la présidence et la vice-présidence du gouvernement du Sud-Soudan, $88 \%$ des postes ministériels et $94 \%$ de ceux de l'assemblée législative, dont la présidence. Il bénéficie en outre de la loyauté du leadership militaire. Fort de cette ébauche de construction hégémonique, le MPLS entend bien prolonger cette situation de « transition » instituée par le CPA au-delà de la sécession de juillet $2011^{22}$.

Pourtant, les défis lancés au MPLS par des Sudistes, avec le soutien de Khartoum, ne manquent pas. Pour lever l'apparente contradiction avec ce qui précède, il faut d'abord voir que les autorités de Khartoum sont traversées par d'importantes contradictions. Le texte de Roland Marchal dans ce dossier met ainsi l'accent sur cette génération de civils arrivée aux postes de responsabilité depuis la fin des années 1990, et qui hésite, selon les parcours de chacun, entre la coopération avec les partenaires sudistes et internationaux pour une paix réussie et la collusion avec les services de sécurité soudanais qui jouent le pourrissement contre le Sud et ses alliés. En outre, on notera que le choix tactique de contribuer à asseoir le MPLS au pouvoir à Juba pour assurer le succès de la sécession n'empêche pas les efforts nordistes destinés à éviter que celui-ci étende son emprise sur des territoires frontaliers contestés et riches en ressources.

18. Voir J. Young, «Sudan: The Incomplete Transition from the SPLA to the SPLM », in J. de Zeeuw (dir.), From Soldiers to Politicians. Transforming Rebel Movements after Civil War, Boulder, Lynne Rienner, 2008, p. 159 .

19. Voir R. Marchal, «Le facteur soudanais, avant et après », Critique internationale, nº 17, 2002, p. 46.

20. L'Igad est constituée de l'Éthiopie, de Djibouti, du Kenya, de l'Ouganda, du Soudan, de la Somalie et de l'Érythrée.

21. Voir J. Young, «Sudan : The Incomplete Transition... », art. cit., p. 165.

22. International Crisis Group, « Politics and Transition.. », doc. cit., p. 2 et 7-9. 


\section{Négocier les attributs du nouvel État : conflits fonciers et décentralisation}

Dans le dossier qu'ils ont dirigé en 2010 dans Development and Change, Tobias Hagmann et Didier Péclard invitaient à observer "par le bas » les pratiques par lesquelles des acteurs et des groupes sociaux différents investissent localement la forme étatique et s'engagent ainsi dans la « négociation » quotidienne de l'État ${ }^{23}$, contribuant dans le même temps à en dessiner les contours selon des ressources et des tempos différents. Les contributions de Naseem Badiey et Lotje de Vries montrent, chacune à sa façon, l'intérêt de ce regard lorsqu'il est appliqué au moment de la transition politique vers l'indépendance. Il permet en effet de saisir sur le vif, avant leur éventuelle ossification, des moments de négociation encore désordonnés relatifs aux attributs mêmes de l'État, ou plutôt aux attributs propres à cet État qui naît de la « situation étatique » (statehood).

Un premier attribut de souveraineté que le gouvernement sud-soudanais négocie âprement depuis 2005 concerne la politique urbaine et foncière au sein de la capitale du futur État indépendant, Juba. Naturellement, ces projets fonciers n'opèrent pas dans un environnement socialement et institutionnellement vierge. Les dynamiques locales contingentes retiennent rarement l'attention des experts du statebuilding, focalisés sur les dispositions conclues et les relations entre anciens belligérants "centraux»- cette négligence obérant les chances de « succès » pour ce dernier. Ces dynamiques résultent de rivalités anciennes entre populations équatoriennes installées de longue date dans la province de Juba (État de l'Equatoria central) et populations dinka qui ont consolidé leurs positions acquises localement depuis les années $1970^{24}$.

La première expérience d'autonomie administrative du Sud-Soudan, à la suite des accords de paix d'Addis-Abeba de 1972, a en effet bénéficié à des Sudistes éduqués, surtout dinka, qui ont investi l'appareil administratif régional à Juba et se sont installés durablement dans la zone. Les rivalités ont été aiguisées par le renversement de situation consécutif au déclenchement de la guerre par l'APLS en 1983 : Juba est alors devenue une ville-garnison de l'armée soudanaise, ce qui facilita les relations entre les Équatoriens et les autorités civiles et militaires nord-soudanaises, tandis que les Dinka engagés dans la lutte armée contre Khartoum au sein de l'APLS durent quitter la zone.

De ce double héritage résultent aujourd'hui des tensions ravivées entre les élites équatoriennes et les anciens commandants dinka de l'APLS devenus des barons du MPLS tensions qui traversent les débats sur les compétences respectives des différentes institutions politiques et administratives situées à Juba. Fortes du discours international valorisant la décentralisation en faveur des « communautés locales », les élites équatoriennes ont largement investi la nouvelle autorité politique et administrative provinciale créée par le CPA, l'État de l'Equatoria central (CES), situé à Juba. L'influence des dynamiques locales intervient ici : la question de l'allocation des terres dans la ville de Juba est devenue une pomme de discorde entre d'une part le CES, soutenu par des franges équatoriennes arrimées à des revendications identitaires fortes (la jeunesse bari par exemple) contre des Dinka accusés de les spolier, et d'autre part le gouvernement du Sud-Soudan, dont les projets d'urbanisme destinés à transformer Juba en capitale moderne d'un État unifié et multiethnique heurtent des intérêts locaux équatoriens. Cependant, cette opposition binaire, fréquente dans la rhétorique des Sud-

23. T. Hagmann et D. Péclard, « Negotiating Statehood: Dynamics of Power and Domination in Africa », Development and Change, vol. 41, $\mathrm{n}^{\circ} 4,2010$, p. 539-562.

24. Voir à ce propos les tensions entre Dinka et Équatoriens toposa dans l'État de l'Equatoria oriental : A. Walraet, «Le Sud-Soudan dans l'attente du référendum. Un regard sur la formation de l'État par le bas », Politique africaine, $\mathrm{n}^{\circ} 119$, octobre 2010, p. 189-205. 
Soudanais, ne doit pas conduire à minimiser les lignes de fracture existant dans les revendications locales pour la terre entre Equatoriens et Nuer, et parmi les Equatoriens euxmêmes.

On ne sait pas si Juba restera le site de cette capitale en projet. L'article de Naseem Badiey informe en tout cas des défis que le nouvel État devra relever en matière de répartition des compétences et, plus essentiellement, en matière de règlement des divisions inter-sudistes persistantes sur fond de compétition foncière aiguë. L'auteure espère que le débat sur l'allocation des terres servira d'apprentissage en vue d'une conciliation des intérêts en présence. Nous le mentionnions plus haut, une orientation plus hégémonique (une autre forme de stabilisation) semble tenter la direction du MPLS dans son rapport à ses "périphéries ». Les observateurs ont rapporté des irrégularités imputées au MPLS lors des élections générales d'avril 2010 (intimidations, ingérences, détentions arbitraires, fraudes alléguées), en particulier dans le CES, précisément ${ }^{25}$. En outre, en raison de la réforme du système électoral, $96 \%$ des députés élus dans cet État pour siéger à l'Assemblée législative du Sud-Soudan concouraient sous la bannière du parti majoritaire, recueillant $71 \%$ des voix exprimées ${ }^{26}$. Des résultats similaires ont marqué les autres provinces du Sud-Soudan ${ }^{27}$.

Un autre attribut en cours de négociation fait l'objet d'un éclairage particulier dans ce dossier : le contrôle des frontières, ou plutôt des marches de l'État sud-soudanais en territoire congolais. Ici encore, l'État ne se décrète pas, mais se négocie dans un contexte contraint. Le caractère transfrontalier de cette question, qui semble se jouer dans la seule relation entre le futur État, sa (future ex-) tutelle et le voisin, n'exclut pourtant pas les jeux politiques intersudistes.

\section{L’État-MPLS : atavisme rebelle et chevauchement des ordres politiques}

Le MPLS est-il capable de produire un ordre politique civil stable ? C'est la question que l'on se pose à la lecture du texte de Lotje de Vries. Elle résonne avec une problématique qui suscite aujourd'hui l'intérêt des spécialistes des conflits armés intra-étatiques et des praticiens de la paix et de la sécurité : celle de la civilianisation des groupes rebelles et de leur transformation en partis politiques ${ }^{28}$. Pour le Sud-Soudan, les éléments de réponse apportés par John Young en 2008 poussent au pessimisme ${ }^{29}$. Des années durant, l'APLS a déployé un gouvernement de guérilla ${ }^{30}$, fondé sur la relation personnelle au chef militaire historique. Depuis Addis-Abeba, Nairobi ou son quartier général de Chukudum, John Garang a travaillé à conserver le mouvement dans un état de sous-institutionnalisation. Il s'agissait de servir la lutte armée la plus efficace possible contre les soldats soudanais (souvent darfouris), et plus encore contre les milices pro-Khartoum des Forces de défense populaires ${ }^{31}$, et non de produire des structures administratives civiles, des programmes politiques ou la cooptation patiente des voix discordantes en faveur d'un large rassemblement populaire. Ceci explique également le niveau de violence que l'APLS a pu exercer contre des civils durant la guerre, en particulier dans les moments de fragmentation du mouvement après l'expulsion d'Éthiopie et la scission

25. Centre for International Governance Innovation, «Southern Sudan », Security Sector Reform Monitor, $\mathrm{n}^{\circ} 4$, janvier 2011 ; International Crisis Group, « Politics and Transition... », doc. cit., p. 2-3.

26. International Crisis Group, « Politics and Transition... », doc. cit., p. 27.

27. Voir A. Walraet, « Le Sud-Soudan dans l'attente... », art. cit.

28. Voir J. de Zeeuw (dir.), From Soldiers to Politicians..., op. cit.

29. J. Young, «Sudan : The Incomplete Transition... », art. cit.

30. Ø. H. Rolandsen, Guerilla Government. Political Change in the Southern Sudan during the 1990s, Uppsala, Afrika Institutet, 2005.

31. Voir J. Salmon, A Paramilitary Revolution : The Popular Defence Forces, Genève, Small Arms Survey/The Human Security Baseline Assessment, 2007. 
entre Garang, d'une part, et Lam Akol et Riek Machar, d'autre part, en 1991. L'APLS n'incarne certes pas à tous égards le modèle de la rébellion dite «opportuniste » proposé par Jeremy Weinstein, dépendant uniquement des ressources minières ou des soutiens captés à l'extérieur, et pouvant de ce fait plus aisément recourir à des violences de masse indiscriminées ${ }^{32}$, mais il en partage certains traits, au premier rang desquels sa forte extraversion ${ }^{33}$.

C'est donc ce mouvement qui contrôle le pouvoir au sein du gouvernement du Sud-Soudan. Garang n'est plus, certes, et son successeur n'a pas montré les mêmes dispositions pour l'autoritarisme charismatique et la personnalisation des liens. Néanmoins, Salva Kiir Mayardit a dû ménager à la fois les anciens commandants compagnons de Garang et son vice-président, Riek Machar, l'homme fort de son gouvernement. Il a consacré l'essentiel de son énergie à unir les forces sudistes rivales pour la victoire du référendum. La mission est accomplie. Avec l'indépendance, et du fait notamment du soutien important qu'il a trouvé sur la scène internationale, cet État sera poussé à se conformer à des modes de gouvernement civils qui peuvent fragiliser localement des élites issues de la guérilla. L'exercice est sensible : l'ancienne garde témoigne de crispations qui facilitent les accès de violence face aux défis politiques et militaires lancés localement par des groupes rivaux.

Parmi ces crispations, il y eut cette journée d'affrontements entre forces du gouvernement sud-soudanais et forces armées de la RDC voisine autour du village de Bazi (Equatoria central) en janvier 2008, telle que la relate Lotje de Vries. À l'occasion de ce clash sans grande gravité, d'anciens « libérateurs » de la zone, luttant au sein de l'APLS contre les forces pro-Khartoum en 1997, ont montré qu'ils ne renonçaient pas à une position localement dominante conquise précisément par ce fait d'armes contre l'ennemi nord-soudanais, en dépit des positions officielles qu'ils occupent aujourd'hui à Juba. En effet, dès 1997, ce "gouvernement de libération" installé par l'APLS dans la zone déborde les frontières congolaises. Depuis, l'APLS s'est toujours refusée à abandonner cette présence militaire en territoire congolais, jusqu'à cet épisode du 24 janvier 2008 débouchant sur une échauffourée frontalière, puis une solution négociée.

On voit, par cette entrée très «micro », combien l'hypersensibilité du futur État aux défis lancés à son autorité renvoie aux statuts localement conquis par des commandants APLS, qui ont su «capitalis[er] la notoriété acquise pendant le conflit et [...] la proximité construite alors avec un territoire et une population de par [leur] propre localisation militaire ${ }^{34} \gg$. Pour l'auteure, deux ordres politiques se chevauchent ici, investis par des groupes localement concurrents, entre d'une part des élites dinka en position de force au sein de l'APLS et aujourd'hui dans les institutions centrales sud-soudanaises à Juba et, d'autre part, des élites issues des communautés équatoriennes, installées de longue date de part et d'autre de la frontière, et qui comptent sur le CPA de 2005 pour renforcer un nouvel ordre politique et administratif civil et décentralisé, au détriment des hiérarchies héritées de la lutte armée. Il ne s'agit donc pas seulement d'ethos et de pesanteurs, mais bien d'enjeux très actuels pour les protagonistes. Cette contribution prolonge l'invitation lancée par Roland Marchal et Christine Messiant à scruter les transformations durables des statuts sociaux, qui perdurent bien après le temps de la guerre ${ }^{35}$.

32. Par opposition à la rébellion dite « activiste », dépendant des ressources captées en vertu d'une relation de solidarité avec son environnement social immédiat. Voir J. M. Weinstein, Inside Rebellion : The Politics of Insurgent Violence, Cambridge, Cambridge University Press, 2007.

33. Voir J. Young, « Sudan : The Incomplete Transition... », art. cit., p. 173-174.

34. N. Duclos, « Introduction », in N. Duclos (dir.), L'Adieu aux armes ? Parcours d'anciens combattants, Paris, Karthala, 2010, p. 37.

35. R. Marchal et C. Messiant, Les Chemins de la guerre et de la paix. Fins de conflits en Afrique orientale et australe, Paris, Karthala, 1997. 


\section{Ajustements régionaux et surveillance internationale}

Dans son article, Roland Marchal retrace de manière systématique les lignes régionales qui se modifient à l'approche de cette sécession sudiste. Cet écheveau régional passe d'abord par Khartoum, l'ancienne tutelle et futur voisin omniprésent de Juba. La politique nordsoudanaise peut connaître demain un bouleversement autour de l'enjeu crucial - sans cesse reporté - de la refonte de la citoyenneté sur l'ensemble du territoire, car le Sud occupait une place peu enviable mais non moins "utile » dans les hiérarchisations existantes entre groupes soudanais ${ }^{36}$. L'élite militaro-islamiste de Khartoum peine en tout cas à surmonter le schisme qu'elle a connu en 1999-2000, conduisant à l'éviction du vieux guide islamiste al-Tourabi ${ }^{37}$, ce qui accélère le tribalisme politique ainsi qu'une certaine radicalisation politique au sein des courants islamistes au Soudan ${ }^{38}$. Dans sa politique à l'égard du Sud, Khartoum gagne en marge de manœuvre du fait des changements en cours en Égypte (qui pourraient renforcer les Frères musulmans, approfondissant des relations déjà amicales depuis 2000) et en Libye (très intéressée par le Darfour), de même que le régime érythréen voit ses capacités de nuisance vis-à-vis de Khartoum se réduire, après avoir joué un rôle de premier ordre.

Plus au sud, le Kenya confirme sa proximité politique et commerciale avec Juba, alors que l'Éthiopie tempère ses sympathies envers le MPLS pour ménager ses partenaires nordsoudanais (en matière d'infrastructures pétrolières et routières). On verra avec la contribution d'Umberto Tavolato à ce dossier que le régime de Meles Zenawi joue des coudes au sein de l'Union africaine pour affirmer son rôle diplomatique de garant de la stabilité régionale - avec un certain succès à Washington et Bruxelles, peut-être moins à Addis-Abeba. Mais il souffre au plan militaire de son engagement face au djihadisme en Somalie. La route semble donc dégagée pour le régime ougandais et son appétit de puissance dans la région ${ }^{39}$, qui passe par le soutien diplomatique, militaire et commercial aux Sud-Soudanais, ainsi qu'aux rebelles darfouris, contre Khartoum. Enfin, l'Igad semble avoir perdu le rôle que les États de la région, en particulier l'Éthiopie, lui avaient reconnu dans les négociations de paix Nord-Sud dès 1993. Khartoum y voit en effet une arène hostile et lui préfère l'Union africaine (UA), tandis que les États de la région privilégient aujourd'hui la Communauté est-africaine.

Les enjeux pétroliers pèsent lourdement dans ces dynamiques régionales et internationales, et hypothèquent l'avenir des relations entre les deux Soudan. Benjamin Augé y consacre sa contribution au dossier. À très court terme, émerge le défi du partage des champs en activité situés en zone frontalière. Ces négociations impliquent le cœur du pouvoir dans les deux camps. Depuis le lancement des activités d'extraction pétrolifère en 1999, le président Béchir

36. Une analyse ancienne mérite ici d’être remémorée : «Les Soudanais éduqués regardent le Sud comme l'Égypte les regarde. La perte du Sud deviendrait une question de prestige et une relative source d'anxiété pour eux, mais pèse également la crainte de découvrir a posteriori des richesses au Sud capables de garantir l'indépendance du Soudan. Comme le Nord Soudan pour l'Égypte, le Sud constitue une source de main d'œuvre et de domestiques bon marché." in Fabian Colonial Bureau, Sudan. The Road Ahead, Londres, Fabian Publications, 1947, repris par J. Oduho et W. Deng, The Problem of the Southern Sudan..., op. cit., p. 17.

37 Dès 1996, Hassan el-Tourabi lance une série de réformes internes qui le replace progressivement au cœur des institutions politiques soudanaises, contre ses lieutenants d'hier qui tiennent l'appareil d'État et le secteur de la sécurité. Ces derniers rompent avec lui et le chassent du pouvoir, l'érigeant en opposant acharné du régime. Cette scission s'inscrit dans un mouvement d'inflexion du régime face à ses activités passées en faveur de mouvements radicaux anti-occidentaux, à la suite des attentats contre les ambassades américaines à Nairobi et Dar es Salaam en 1998 et des ripostes décidées contre lui (sanctions et bombardement américain de l'usine d'al-Shifa). R. Marchal, « Le Soudan d'un conflit à l'autre », Les Études du CERI, n 107-108, 2004.

38. Voir aussi E. Ahmed, «L'islam politique... », art. cit. Merci également à Jago Salmon pour ses indications sur ce point.

39. Voir S. Perrot, « Les nouveaux interventionnismes militaires africains. Une redéfinition des conditions de la puissance au sud du Sahara? », Politique africaine, $n^{\circ}$ 98, juin 2005, p. 111-130. 
et son entourage ont pris le contrôle des échanges entourant cette activité pétrolière, via le ministère du Pétrole et la Sudapet ${ }^{40}$. Depuis 2005, ce contrôle présidentiel reste tenace. Au Sud-Soudan, dans un gouvernement dont le budget est issu à $98 \%$ de la rente pétrolière, le MPLS envisage avec espoir le développement des relations avec les grands acteurs du secteur, chinois notamment. Pour l'heure cependant, les compétences manquent au Sud.

Du fait des infrastructures actuelles, qui dirigent le brut du Sud exclusivement vers le Nord, vers Khartoum puis Port-Soudan, le Sud-Soudan ne pourra gagner immédiatement en autonomie vis-à-vis du Nord. La construction d'oléoducs alternatifs en direction du Kenya dépendra des résultats des forages actuels dans l'Ouganda voisin, mais aussi des résultats obtenus dans le bloc B au Sud-Soudan, si Total parvient à trouver le partenaire tant recherché... Le Nord considère également des projets de nouveaux oléoducs. Tout cela pourrait favoriser un éloignement entre Nord et Sud. Pas dans l'immédiat, toutefois : Juba ne peut qu'acheter le produit raffiné à Khartoum, ce qui pose la question du prix auquel il entend vendre à ce dernier son brut. L'interdépendance actuelle, évidente dans ce domaine ou pour le partage des eaux du Nil (voir la contribution de Roland Marchal) dans des cadres davantage multilatéraux, n'empêche pourtant pas les pics de tensions que nous connaissons aujourd'hui dans les zones frontalières contestées.

A propos du rôle des institutions multilatérales pour le Sud Soudan, la contribution d'Umberto Tavolato nous plonge précisément au cœur des options discutées à l'UA à l'égard de la sécession en cours, entre réticences de principe et opportunités nouvelles pour l'organisation continentale. L'auteur, lui-même praticien en poste à Addis-Abeba, a observé le travail du panel de haut niveau créé en 2009 par l'UA autour de Thabo Mbeki, et mesuré à travers lui le poids de l'héritage reçu de l'OUA - le principe d'inviolabilité des frontières africaines, mais aussi la faiblesse des relations avec les anciens rebelles de l'A/MPLS. Les membres de l'organisation panafricaine ont toutefois choisi d'investir ce dossier médiatisé pour ne pas le laisser au seul leadership de l'ONU ou des États-Unis et ainsi affirmer le nouveau rôle auquel l'organisation aspire en matière de règlement diplomatique des conflits sur le continent. Pour ce faire, il aura fallu l'entregent de Meles Zenawi au sein de l'UA, ainsi que les fortes tensions provoquées en Afrique par les procédures engagées par la Cour pénale internationale sur le continent (au Soudan en particulier), sans oublier le choix du président Béchir de mobiliser l'UA pour mieux rejeter l'Igad et réfréner l'ONU.

Une fois mobilisé, le panel de haut niveau a su faire évoluer ses positions de principe et ses préférences pour prendre en compte les obstacles politiques les plus immédiats à toute solution négociée, en particulier la détermination farouche du SPLM à ne pas se laisser enfermer par la promesse d'un futur «nouveau Soudan » uni et démocratique.

L'inéluctabilité de la sécession a finalement imprégné le jeu politique au sein de l'UA et le discours produit par le panel de haut niveau, dès lors qu'elle faisait figure d'exception propre au Sud-Soudan et pouvait être présentée comme l'achèvement d'un processus engagé en 1946 et avorté en 1956. Par cette prise de position, l'UA n'a certes pas révolutionné le traitement international de ce dossier. Elle n'en a pas moins fait progresser ses propres marges de manœuvre dans le domaine de la paix et de la sécurité sur le continent, en dépit des réticences initiales d'États membres très influents. L'accord de cessez-le-feu du 20 juin dernier concernant Abyei (évoqué ci-dessus) obtenu par le panel de haut niveau de l'UA confirme en tout cas le volontarisme des acteurs africains dans ce dossier.

L'article d'Umberto Tavolato renvoie donc aux acteurs multilatéraux de la régulation internationale des conflits armés sur le continent. Il illustre notamment certains enjeux et certaines modalités de l'exercice du leadership diplomatique au sein de ces organisations en matière de paix et de sécurité ${ }^{41}$.

40. La Sudapet, société d'État, possède des parts dans tous les blocs exploités au Soudan.

41. À propos de cette question du leadership en contexte de gestion multilatérale des conflits armés, voir 
Impossible, en effet, de ne pas évoquer cette importante présence internationale au Soudan dans le succès du référendum, mais aussi dans la « gestion » de la question darfourie, c'est-àdire le gel plus ou moins efficace des positions des belligérants et le maintien précaire des déplacés à l'écart des violences. Plus de 35000 personnels en uniforme servant deux missions de l'ONU - la Minus (Mission des Nations unies au Soudan) et la Minuad (Mission des Nations Unies et de l'Union Africaine au Darfour), opération conjointe avec l'UA après l'échec de la mission de cette dernière de 2004 à 2007 - sillonnent le territoire, un chiffre à comparer aux 100000 personnels en uniforme aujourd'hui mobilisés pour l'ensemble des opérations de l'ONU à travers le globe. De nombreux travaux académiques sur les actions pacificatrices internationales portent désormais sur le Soudan (essentiellement sur le Darfour, vu son importance médiatique dans les pays occidentaux), terrain privilégié pour tester la fameuse « responsabilité de protéger ${ }^{42}$. Le Soudan et l'imbrication entre Sud-Soudan et Darfour offrent un terrain utile pour qui veut sonder les pratiques des professionnels internationaux de la paix et de la sécuritét ${ }^{43}$, et les opportunités d'extraversion que ces pratiques internationales génèrent ${ }^{44}$.

En l'occurrence, il est difficile de ne pas lier l'engagement international, progressivement promu par l'administration américaine dès 2001, au soutien que John Garang a trouvé auprès du département d'État au cours des années 1990 (par le truchement d'organisations chrétiennes américaines) pour compenser le retrait par l'Éthiopie de son soutien à l'APLS en $1991^{45}$. Ce cas rappelle l'importance de cette quête d'extraversion de la part non seulement des États militairement faibles, mais aussi des groupes armés rebelles, ce qui fait dire à Christopher Clapham que ces acteurs recherchent - plus qu'ils ne subissent - les relations de patronage international ${ }^{46}$.

Au moment où nous écrivons ces lignes, le MPLS va probablement réussir son pari : bénéficier de ce puissant acte performatif par lequel les États de la planète exhausseront le gouvernement autonome du Sud Soudan au rang de souverain. La fenêtre d'opportunité pour atteindre ce résultat aura été si étroite que le MPLS de Salva Kiir y aura concentré tous ses efforts, avec cette crainte de chaque instant que le «partenaire » nordiste ait envie et, surtout, trouve les moyens d'interrompre le processus. C'est cette route vers l'indépendance et la souveraineté, une route particulièrement escarpée et pourtant pensée comme une solution pacificatrice entre le Nord et le Sud, que le présent dossier vient éclairer.

David Ambrosetti

CNRS - ISP - Université Paris Ouest

D. Ambrosetti, Normes et rivalités diplomatiques à l'ONU. Le Conseil de sécurité en audience, Bruxelles, P.I.E. Peter Lang, 2009, chapitre 5.

42. Citons à titre d'exemple R. Barltrop, Darfur and the International Community: The Challenges of Conflict Resolution in Sudan, Londres, Tauris Academic Studies, 2011; P. D. Williams et A. J. Bellamy, "The Responsibility to Protect and the Crisis in Darfur», Security Dialogue, vol. 36, n 1, 2005, p. 27-47; M. M. Gabrielsen, «La sécurité humaine et l'internationalisation des conflits intra-étatiques : le cas du conflit au Sud-Soudan », Revue de la sécurité humaine, $\mathrm{n}^{\circ}$ 3, 2007, p. 29-42.

43. D. Ambrosetti, "Urgences et normalités de gestionnaires face aux violences "des autres" : l'ONU et le Soudan », Actes de la recherche en sciences sociales, $\mathrm{n}^{\circ}$ 174, 2008, p. 81-99.

44. A. de Waal, "Mission without end? Peacekeeping in the African political marketplace", International Affairs, vol. $85, \mathrm{n}^{\circ} 1,2009$, p. 99-113.

45. J. Young, « Sudan : The Incomplete Transition... », art. cit., p. 163-164 et 173-175.

46. C. Clapham, Africa and the International System..., op. cit., chapitre 9. 